\title{
Six-Month Post-Acute COVID-19: High Self-Reported Morbidity Among Adults Younger Than Sixty Years and Females
}

\author{
Ashish Bhargava ${ }^{\mathrm{a}, \mathrm{b}}$ (D), Wei Zhao ${ }^{\mathrm{a}}$, Mamta Sharma ${ }^{\mathrm{a}}$, \\ Susanna M. Szpunar ${ }^{\mathrm{a}}$, Louis Saravolatz ${ }^{\mathrm{a}}$
}

\section{To the Editor}

The outbreak of novel coronavirus disease 2019 (COVID-19) has infected over 254 million people, causing 5.1 million deaths worldwide. Symptomatic COVID-19 patients who present with acute hypoxemic respiratory failure require hospital care where acute lifesaving measures can be implemented. The health consequences, however, go well beyond hospitalization [1]. Longterm sequelae across multiple medical domains, including the respiratory, psychiatric, infectious, rheumatologic, and neurocognitive have been reported after COVID-19 [2,3]. Prevalence of these long COVID conditions vary from 5\% to $80 \%$ depending upon the case definitions and population studied [4].

We aimed to describe the self-reported occurrence of symptoms and their effect on patient's functioning 6 months after their acute hospitalization for COVID-19.

From a historical cohort study of hospitalized COVID-19 patients in our hospital, we identified patients discharged home, between March 8 and June 14, 2020 [5]. COVID-19 was confirmed via reverse transcriptase-polymerase chain reaction assay of a nasopharyngeal swab. Patients were contacted by telephone 6 months after discharge. The purpose of the study was explained, and they were asked to consent to a telephone questionnaire. We used a modified version of a previously validated general symptom questionnaire (GSQ-30) to assess multi-system symptom burden among patients with post-treatment Lyme disease syndrome [6]. It is easy to administer and strongly correlates with the functional impairment among patients with multisystem disease. The Patient Health Questionnaire-2 (PHQ-2) was also used to screen for major depression. GSQ-30 questionnaire measures somatic, neurological and neuropsychiatric symptom burden. Each question had five options: "not at all", "a little bit", "somewhat", "quite a bit", and "very much", which

Manuscript submitted November 19, 2021, accepted December 21, 2021 Published online December 28, 2021

aDepartment of Internal Medicine, Thomas Mackey Center for Infectious Disease Research, Ascension St. John Hospital, Detroit, MI 48236, USA

${ }^{b}$ Corresponding Author: Ashish Bhargava, Department of Internal Medicine, Thomas Mackey Center for Infectious Disease Research, Ascension St. John Hospital, Detroit, MI 48236, USA. Email: Ashish.bhargava@ascension.org

doi: https://doi.org/10.14740/jocmr4640 during analysis were converted into "yes" and "no" categories. Category "no" reflected option, "not at all". Demographic and clinical characteristics were available from the original study. The study was approved by the Institutional Review Board.

Of the original 565 patients, 258 patients were discharged home $(45 \%)$ and had a telephone number on record. Of these, $57(22 \%)$ patients were able to be contacted and agreed to participate in the survey. The mean (standard deviation (SD)) age of the respondents was 55.1 (14.8) years, $50(87.7 \%)$ were black, and $37(64.9 \%)$ were female. Of these patients, 37 $(64.9 \%)$ patients had hypertension, $24(42.1 \%)$ had diabetes and $43(75.4 \%)$ were obese. Pneumonia was diagnosed in 35 (61.4\%) patients at the index hospitalization. The most common symptoms at the time of follow-up were fatigue $(60.0 \%)$, dyspnea $(57.1 \%)$, feeling irritable, sad or decreased pleasure $(56.4 \%)$, and memory difficulty $(56.4 \%)$. The mean (SD) GSQ-30 score for the cohort was 30.1 (25.1). Females had a significantly higher mean (SD) GSQ-30 score than males (35.1 (26.5) versus 20.2 (19.3), $\mathrm{P}=0.02)$.

We further stratified our cohort based on their age (Table 1) and sex (Table 2). Mean (SD) GSQ-30 score among patients ages $<60$ year was 29.1 (25.4) compared to 32.1 (25.1), $\mathrm{P}=$ 0.68 among patients ages $\geq 60$ years. Patients ages $<60$ years tended to experience similar, if not worse, impaired functioning $(\mathrm{P}=0.07)$ compared with those ages 60 years and above. Patients ages $\geq 60$ years had more balance problems $(\mathrm{P}=0.02)$. Severity of illness, need for intubation or intensive care unit (ICU) stay and length of hospitalization at the time of index hospitalization were not statistically different among patients ages less than 60 years compared to those over 60 years and above. Females were more vulnerable to the symptoms of fatigue, neurologic and neuropsychiatric domains than males (Table 2). Females had an increased incidence of needing more sleep than usual $(\mathrm{P}=0.05)$, not feeling rested on awakening ( $\mathrm{P}$ $=0.04)$, shooting, stabbing and burning pain $(\mathrm{P}=0.02)$, discomfort with normal light and sound $(\mathrm{P}=0.04)$, feeling irritable or sad $(\mathrm{P}=0.007)$, feeling panicky, anxious or worried $(\mathrm{P}=$ $0.001)$, and memory difficulty $(\mathrm{P}=0.03)$ than males.

Our study describes the clinical burden of post-acute COVID-19 (PAC-19) in four core domains: fatigue, neurological, neuro-psychiatric and viral-like symptoms using GSQ-30 questionnaire. GSQ-30 had been previously validated to assess the functional impairment post-treatment Lyme disease syndrome. Our findings, like previous studies, showed fatigue, and neu- 
Table 1. Self-Reported Post-Acute COVID Syndrome in Adults Younger Than 60 Versus Adults at or Older Than 60 Years

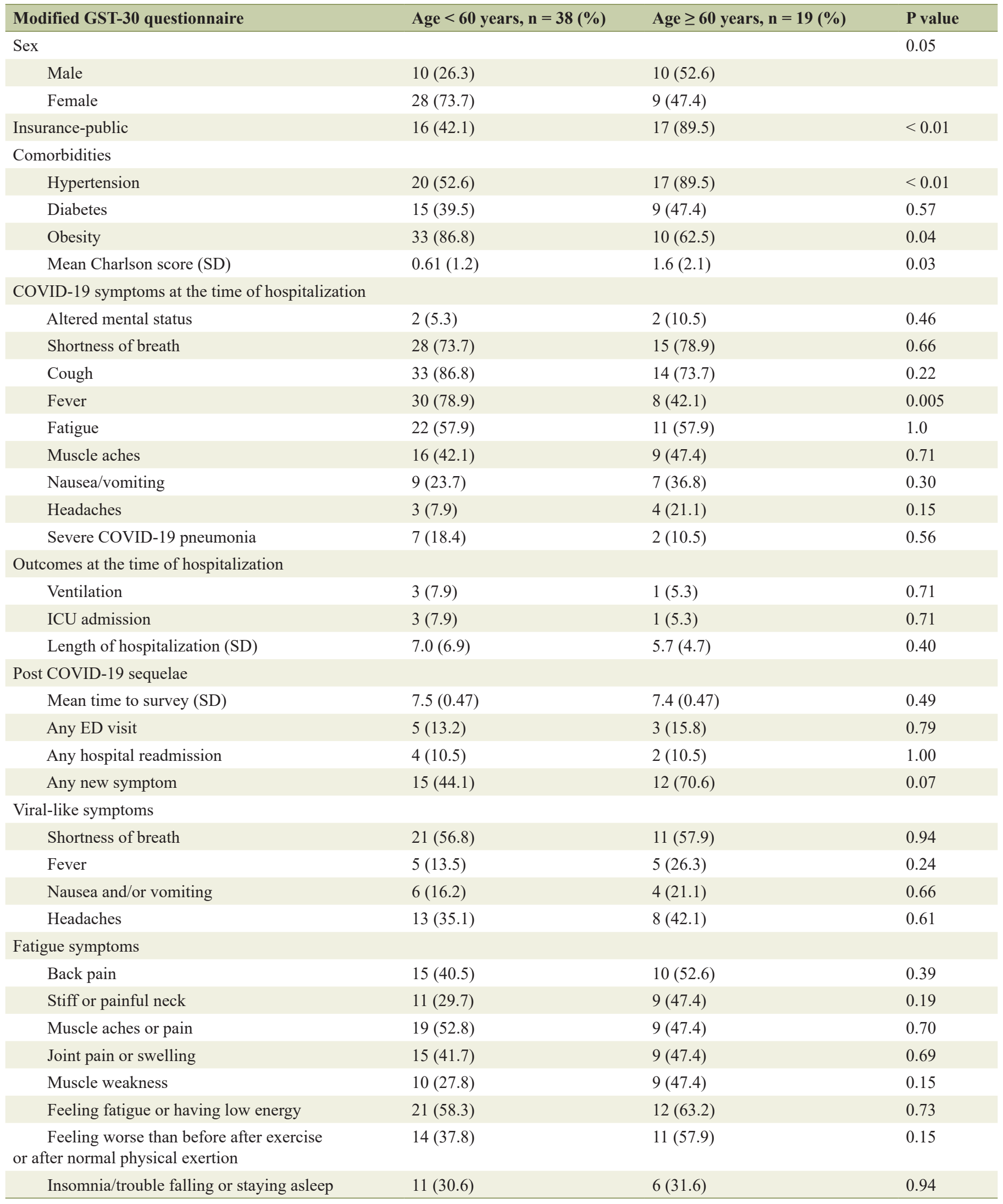


Table 1. Self-Reported Post-Acute COVID Syndrome in Adults Younger Than 60 Versus Adults at or Older Than 60 Years - (continued)

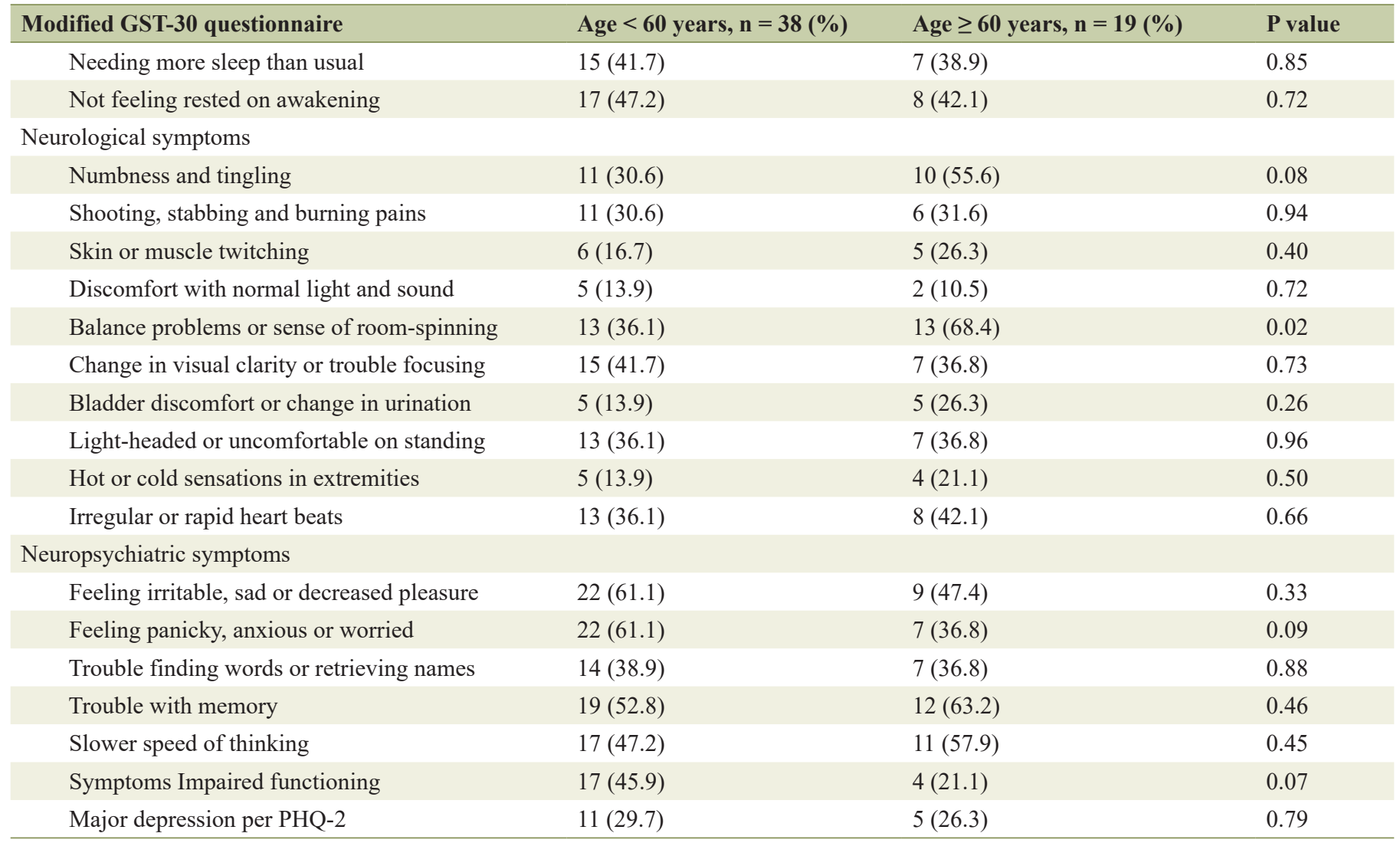

COVID-19: coronavirus disease 2019; SD: standard deviation; ICU: intensive care unit; ED: emergency department; PHQ-2: Patient Health Questionnaire-2.

Table 2. Self-Reported Post-Acute COVID Syndrome in Female Versus Male Adults

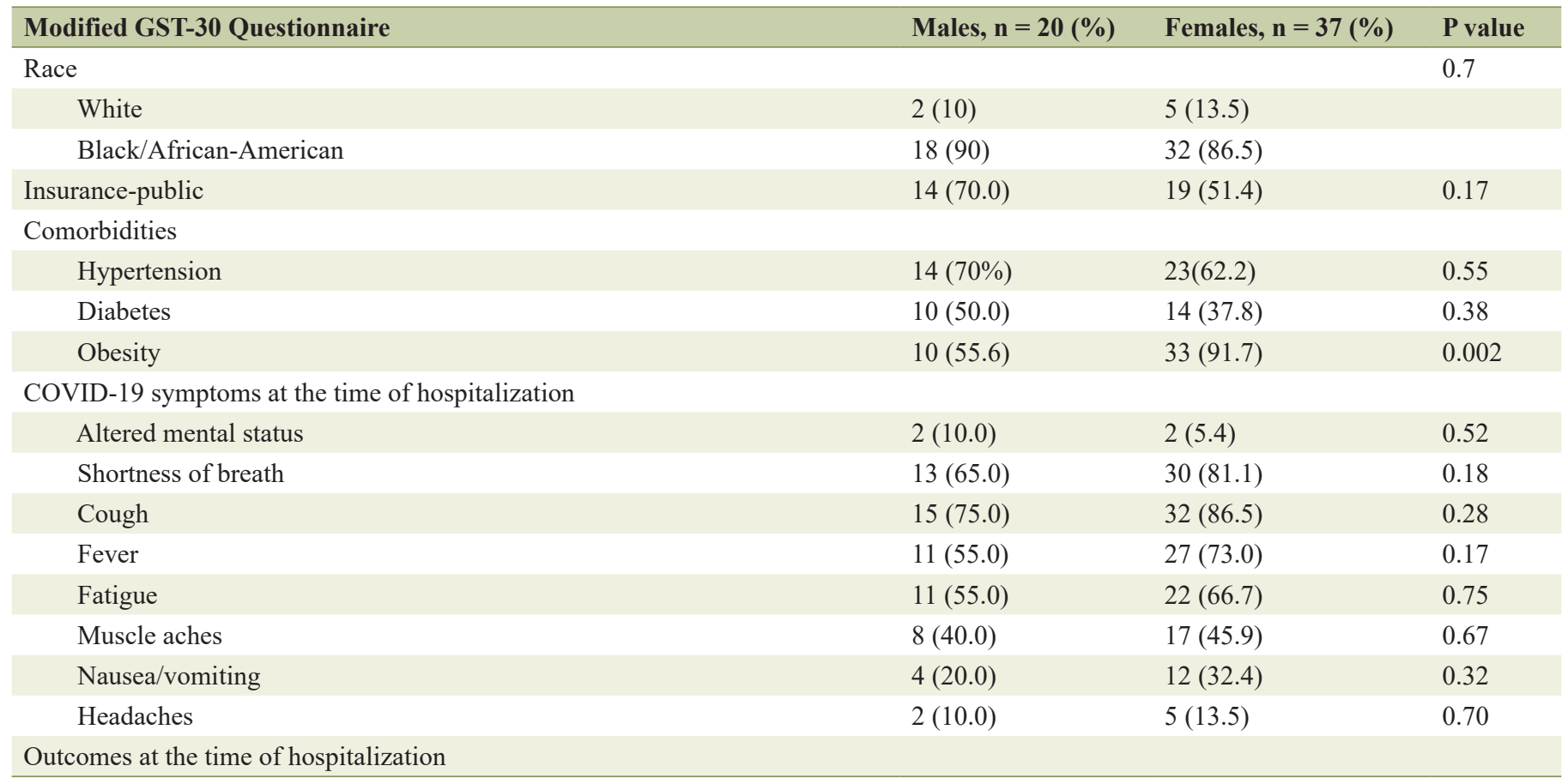


Table 2. Self-Reported Post-Acute COVID Syndrome in Female Versus Male Adults - (continued)

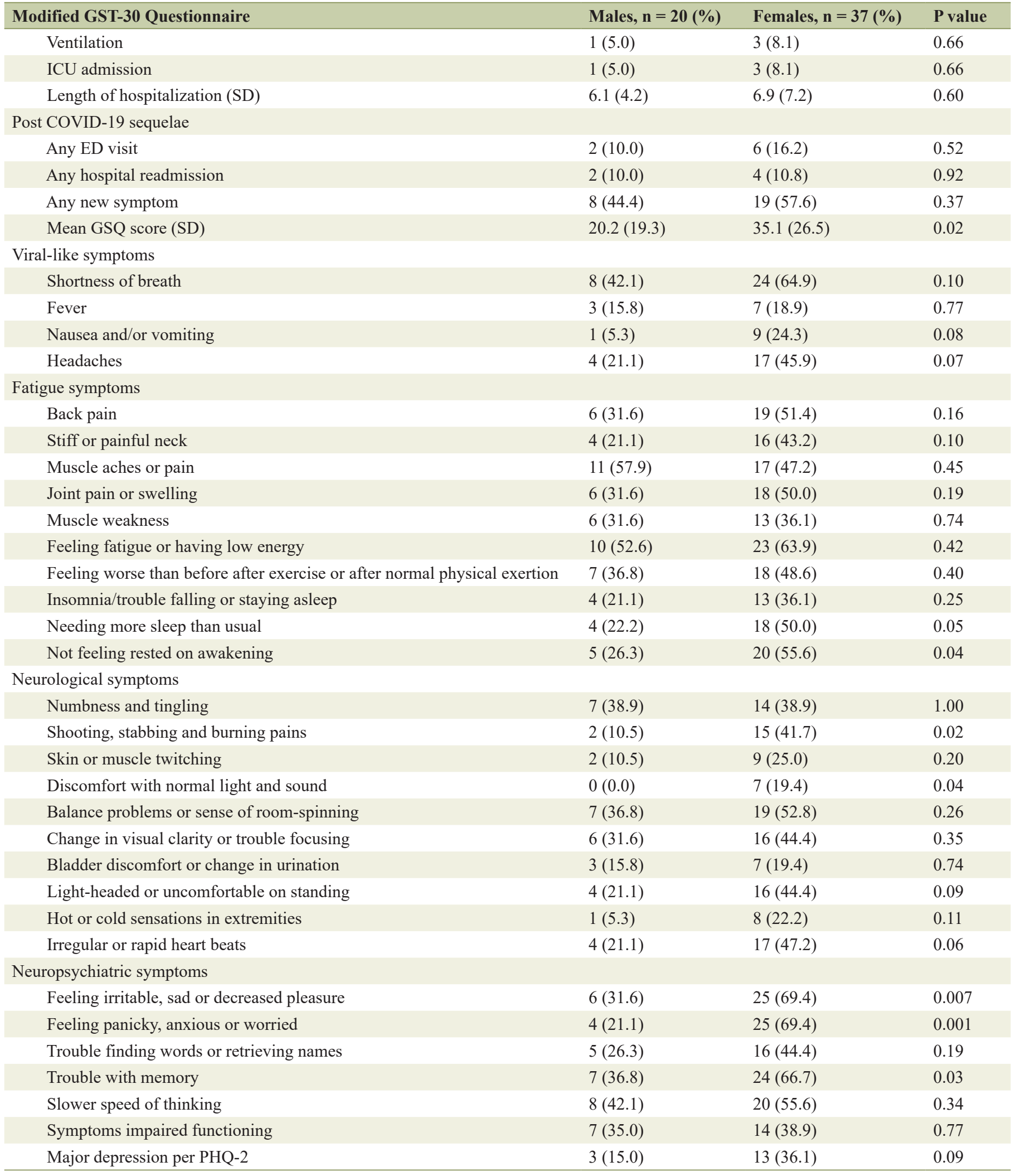

COVID-19: coronavirus disease 2019; SD: standard deviation; ICU: intensive care unit; ED: emergency department; GSQ: General Symptom Questionnaire; PHQ-2: Patient Health Questionnaire-2. 
ropsychiatric symptoms as the most common complaints in COVID-19 survivors [2, 7]. Notably, the younger participants reported significant morbidity, if not worse, than older patients. Over $45 \%$ of patients ages $<60$ years suffered impaired functioning, compared with $21.1 \%$ of patient's ages 60 years and above. This difference approached statistical significance, but larger studies can validate these results. Therefore, early rehabilitation may be targeted for all the COVID-19 survivors.

Females have been reported to be disproportionally affected by psychiatric sequela $[7,8]$. Women and severity of illness has been described as predictors for persistent psychological symptoms [9]. Higher levels of anxiety and depression have been reported earlier among COVID-19 survivors [3, 7, 9]. In our study, females more likely felt sad, irritable, anxious and troubled with memory problems. Our study findings of significantly higher GSQ-30 scores in females compared to men strongly correlates with the functional impairment among the females. Thus, there is utmost urgency to develop a framework and strategy to address this post infectious condition which is causing a great degree of morbidity among younger and female COVID-19 survivors [10].

This study was limited by the relatively low response rate and lack of comparative baseline data on depression. The low response rate, however, was primarily because of not being able to contact people, versus people not agreeing to the survey. Many people who responded to the survey expressed gratitude that the hospital had reached out to see how they were doing. Nevertheless, we provide new information to better understand the full spectrum of PAC-19. Larger studies are needed to further validate our findings.

\section{Acknowledgments}

We thank Ms. Debbie Cicchini for helping us to access the articles from medical journals through the electronic library.

\section{Financial Disclosure}

This research did not receive any specific grant from funding agencies in the public, commercial, or not-for-profit sectors.

\section{Conflict of Interest}

We presented this work at ID week 2021 as poster presentation.

\section{Informed Consent}

Not applicable.

\section{Author Contributions}

$\mathrm{AB}$ contributed to the study design, analysis and manuscript writing. WZ contributed to the preparation of data and manuscript preparation. MS contributed in study design, and manuscript editing. SS did data analysis, and manuscript preparation and editing. LS contributed in study design, and manuscript editing.

\section{Data Availability}

Any inquiries regarding supporting data availability of this study should be directed to the corresponding author

\section{Reference}

1. Bellan M, Soddu D, Balbo PE, Baricich A, Zeppegno P, Avanzi GC, Baldon G, et al. Respiratory and psychophysical sequelae among patients with COVID-19 four months after hospital discharge. JAMA Netw Open. 2021;4(1):e2036142.

2. Logue JK, Franko NM, McCulloch DJ, McDonald D, Magedson A, Wolf CR, Chu HY. Sequelae in adults at 6 months after COVID-19 infection. JAMA Netw Open. 2021;4(2):e210830.

3. Nasserie T, Hittle M, Goodman SN. Assessment of the frequency and variety of persistent symptoms among patients with COVID-19: a systematic review. JAMA Netw Open. 2021;4(5):e2111417.

4. Wanga V, Chevinsky JR, Dimitrov LV, Gerdes ME, Whitfield GP, Bonacci RA, Nji MAM, et al. Long-term symptoms among adults tested for SARS-CoV-2 - United States, January 2020-April 2021. MMWR Morb Mortal Wkly Rep. 2021;70(36):1235-1241.

5. Bhargava A, Szpunar SM, Sharma M, Fukushima EA, Hoshi S, Levine M, Gandhi N, et al. Clinical features and risk factors for in-hospital mortality from COVID-19 infection at a Tertiary Care Medical Center, at the onset of the US COVID-19 pandemic. J Intensive Care Med. 2021;36(6):711-718.

6. Fallon BA, Zubcevik N, Bennett C, Doshi S, Rebman AW, Kishon R, Moeller JR, et al. The general symptom questionnaire-30 (GSQ-30): a brief measure of multi-system symptom burden in Lyme disease. Front Med (Lausanne). 2019;6:283.

7. Taquet M, Geddes JR, Husain M, Luciano S, Harrison PJ. 6-month neurological and psychiatric outcomes in 236379 survivors of COVID-19: a retrospective cohort study using electronic health records. Lancet Psychiatry. 2021;8(5):416-427.

8. Sudre CH, Murray B, Varsavsky T, Graham MS, Penfold RS, Bowyer RC, Pujol JC, et al. Attributes and predictors of long COVID. Nat Med. 2021;27(4):626-631.

9. Huang C, Huang L, Wang Y, Li X, Ren L, Gu X, Kang $\mathrm{L}$, et al. 6-month consequences of COVID-19 in patients discharged from hospital: a cohort study. Lancet. 2021;397(10270):220-232.

10. Phillips S, Williams MA. Confronting our next national health disaster - Long-Haul Covid. N Engl J Med. 2021;385(7):577-579. 